\title{
Analysis of Liquid Piston Compressibility in the Two-Stage Hybrid Positive-Displacement Machine
}

\author{
V. E. Shcherba ${ }^{1 *}, A . V$. Zanin $^{1}$ and E. A. Pavlyuchenko ${ }^{1}$ \\ ${ }^{1}$ Omsk State Technical University, 11, Mira ave., 644050, Omsk, Russia
}

\begin{abstract}
The effect of liquid compressibility in the two-stage piston hybrid positive-displacement machine with a liquid piston was considered. Based on the volume conservation equation, we developed a calculus methodology for the liquid compressibility during gas compression in the second stage of the compressor section. A numerical experiment made it possible to establish that some parameters have the greatest effect on the relative decrease in the height and velocity of the liquid layer. These parameters are the degree of pressure ratio; pipeline length; the polytropic index of the compression; the number of crankshaft revolutions. We found that the relative decrease in the liquid layer height is within $1 \%$, and the relative decrease in the liquid piston velocity is within $5 \%$. The paper analyses the liquid piston compressibility influence on the thermodynamic efficiency of the compression process and compressor performance.
\end{abstract}

\section{Introduction}

One of the cardinal ways to improve the efficiency and weight-size parameters of compressors and positive-displacement pumps is to combine them into a single unit. Such a unit is called the hybrid positive-displacement machine [1,2]. Conducted theoretical $[3,4]$ and experimental studies $[5,6]$ showed the result of their combination:

- improved the compressed gas cooling;

- $\quad$ eliminated compressed gas leakage in the cylinder-piston seal;

- improved cylinder-piston group lubrication and the friction work;

- eliminated dead space in the compressor [7];

- the positive suction head increased;

- the number of moving parts is reduced.

Improving the compressed gas cooling and eliminating compressed gas leakage in the cylinder-piston seal leads to an increase in indicator efficiency [8]. In turn, the elimination of dead space in the compressor and gas leakage in the cylinder-piston seal leads to an increase in the compressor delivery rate. Decreasing friction work in the cylinder-piston group leads to an increase in total efficiency. Decreasing the number of moving parts generally leads to an increase in machine reliability rate and an improvement in weight-size parameters [8].

* Corresponding author: scherba_v_e@ @list.ru 
It should be noted that one piston provides compression and movement of both the compressed gas and the droplet liquid in the piston hybrid positive-displacement machine.

The liquid is used as a piston (liquid piston) for compressing gas to medium and high pressures as the last booster stages. It ensures the liquid supply to the compressor stage by the pump $[9,10]$.

The piston hybrid positive-displacement machine that generates compressed gas and liquid under pressure is ideal for creating the piston hybrid positive-displacement machine for compressing gas to medium and high pressures.

In accordance with [11], the compressed gas enters the compressor section of the second stage from the compressor section of the first stage through interstage pipelines (figure 1). The liquid is supplied to compress gas from the first stage of the pump section to the second stage of the compressor section through the connecting pipe. The operation of such a unit is possible due to the fact that the supply of gas and liquid passes in anti-phase in the first stage of the hybrid machine.

\section{Theory and methods}

The work [12, 13 and others] are devoted to the working processes calculation of the piston hybrid positive-displacement machine (PHPDM).

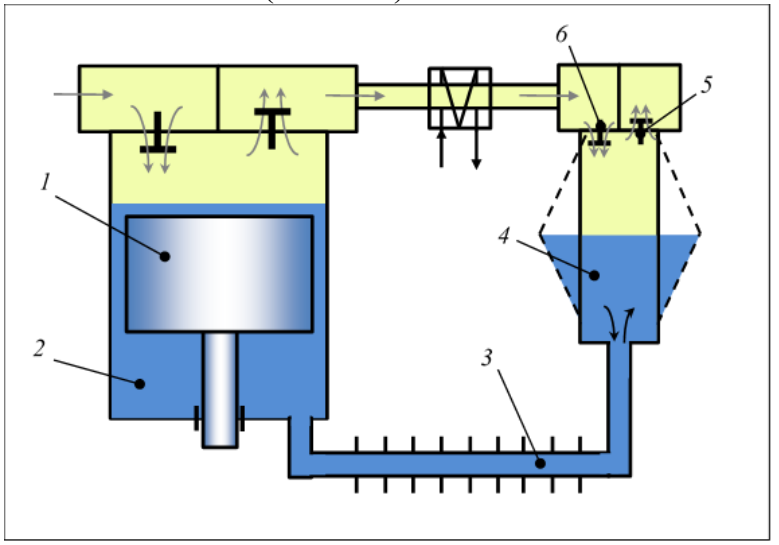

Fig. 1. Diagram of the piston hybrid positive-displacement machine for compressing gas to medium and high pressures

The liquid volume conservation equation used to calculate liquid motion under the assumption of its incompressibility:

$$
Q=Q_{1}=Q_{2}=Q_{i}=\text { const }
$$

In this case, the liquid velocity in any section of the liquid path, and especially at the boundary surface between the compressed gas and the liquid in the second stage, is defined by:

$$
v_{i}=\frac{v_{a} f_{a}}{f_{i}}=\frac{Q}{f_{i}},
$$

where $v_{i}$ is piston velocity, $f_{a}$ is the first stage pump chamber cross-section area, $f_{i}$ is the ith cross-section area.

It should be noted that gas pressure forces, inertia forces, and friction forces act on the liquid, leading to its compression. 
In this case, equation (2) should be expressed taking into account compressibility in the following form:

$$
v_{i}=\frac{(Q-\Delta Q)}{f_{i}}=\frac{\left(v_{a}-\Delta v_{a}\right) f_{a}}{f_{i}},
$$

where $\Delta Q$ is a volume flow rate reduction due to liquid compressibility, $\Delta v_{a}$ is a piston velocity reduction in the first stage pump section due to liquid compressibility.

The compression process calculation in a positive-displacement pump in the presence of a gas cavity was carried out in $[14,15]$. And the liquid and gas compressibility in all processes of the piston pump cycle were taken into account in $[16,17]$.

In carrying out the above studies, friction and inertia forces were neglect because of their modest values. The compressible liquid volumes are significant in the two-stage PHPDM. It is advisable to consider the liquid compressing processes (liquid piston) taking into account inertial and friction forces. We transformed the schematic diagram of the two-stage PHPDM into the design circuit to simplify the calculations (figure 2).

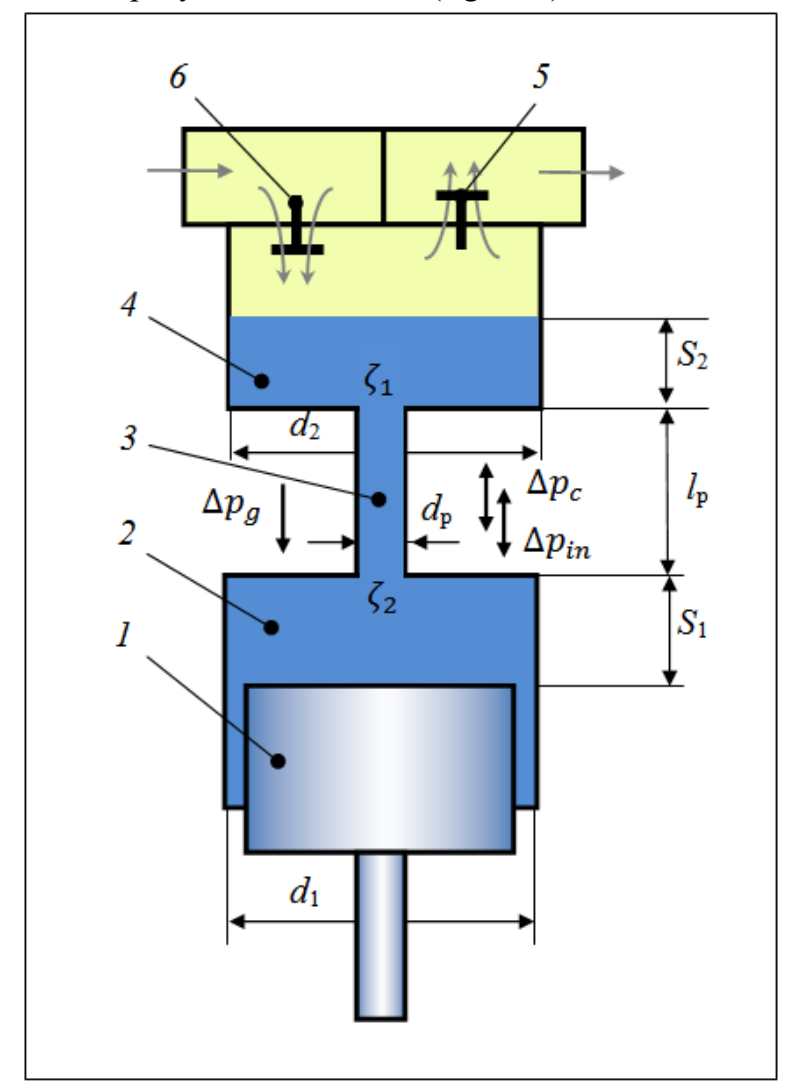

Fig. 2. Design circuit for a liquid compressibility analysis in the two-stage PHPDM.

The liquid from the first stage pump chamber 2 enters the second stage compression chamber 4. It flows through the pipeline 3 when the piston 1 moves up.

A liquid amount increase in the cavity 4 reduces the gas volume, which leads to gas compression and a pressure increase. The discharge valve 5 opens, the compressed gas supplies to the discharge cavity until the gas pressure reaches the discharge pressure. Then compressed gas goes to the gas consumer. 
The liquid from the working cavity 4 moves into the cavity 2 . It flows through the pipeline 3 when the piston 1 moves down.

The gas pressure in the cavity 4 decreases, then the suction valve 6 opens, and the gas enters the cavity 4 from the first stage.

The working liquid in the machine compresses in the processes of compression and discharge. And it expands in the processes of expansion and suction. It is advisable to consider the working liquid compressibility during the gas compression in the second stage of the PHPDM (in the cavity 4). Because of the liquid compression maximum force is due to the gas pressure, or rather, its change.

The change in the working liquid volume in the machine due to the action of gas pressure forces, inertia forces, and friction forces on it:

$$
\Delta V_{w \Sigma}=\Delta V_{w g}+\Delta V_{w i n}+\Delta V_{w f r}
$$

We carried out a sequential consideration of the quantities included in the equation (4).

Liquid volume reduction magnitude $\Delta V_{w \Sigma}$ can be expressed when pressure $\Delta p$ acts on the liquid volume $V_{w}$ from Hooke's law by:

$$
\Delta V_{w \Sigma}=\frac{V_{w} \cdot \Delta p}{E_{w}}
$$

where $E w$ is a bulk modulus of liquid.

The volume conservation equation in the gas compression, including an elementary change in the liquid volume $\Delta V w \Sigma$ and an elementary change in the compressible gas volume, can be expressed as:

$$
\Delta V_{\text {mech }}=\Delta V_{w g}+\Delta V_{w f r}+\Delta V_{w i n}+\Delta V_{g}=\Delta V_{w \Sigma}+\Delta V_{g}
$$

where $\Delta V$ mech is an elementary change in the liquid volume due to the piston 1 movement by the time $\Delta \tau, \Delta V w f r$ is an elementary change in the liquid volume due to the change of the friction forces, $\Delta V w g$ is an elementary change in the liquid volume due to the change of the gas pressure, $\Delta V$ win is an elementary change in the liquid volume due to the change of the inertial forces act on a liquid, $\Delta V g$ is an elementary change in the compressing liquid volume in the cavity 4.

The total liquid volume $\mathrm{Vw}$ includes the liquid volume in the working cavity 4, pipeline 3 , and working cavity 2 .

An elementary change in the liquid volume due to the piston 1 movement can be expressed as:

$$
V_{\text {mech }}=v_{a} f_{p} \cdot \frac{\Delta \varphi}{\omega}
$$

where $v_{a}=\frac{S_{h 1}}{2} \cdot \omega \cdot\left(\sin \varphi+\frac{\lambda}{2} \sin 2 \varphi\right), S_{h 1}$ is a full-length piston stroke in the working cavity 2, $\omega$ is an angular velocity, $\varphi$ is an angle of rotation of the crankshaft, $\lambda=S_{h 1} / 2 l_{p r}$ is the ratio of the piston stroke $\left(S_{h l}\right)$ to the double length of the piston-rod $\left(l_{p r}\right), \Delta \varphi$ is an elementary change of an angle of rotation, $f_{p}=\frac{\pi d_{1}^{2}}{4}$ is a piston area, $d_{l}$ is a piston diameter of the first stage.

Value $\Delta V_{w g}$ can be expressed as:

$$
\Delta V_{w g}=\frac{V_{w}}{E_{w}} \cdot \Delta p_{g}
$$


Gas pressure variation value $\Delta p_{g}$ acting on the liquid is determined based on the use of a polytropic gas compression process:

$$
\Delta p_{g}=p_{i}\left[\left(\frac{V_{g i}}{V_{g i}-\Delta V_{g}}\right)^{n}-1\right]
$$

where $p_{i}$ is a current gas pressure, $V_{g i}$ is a current gas volume, $n$ is the polytropic index of the compression.

An elementary change in the liquid volume due to the change of the inertial forces act on a liquid can be expressed as:

$$
\Delta V_{w i n}=\Delta V_{w i n 1}+\Delta V_{w i n p}+\Delta V_{i n 2}
$$

where $\Delta V_{w i n}=\frac{V_{w 1}}{E_{w}} \Delta p_{i n 1}$ is a change in the liquid volume due to the change of the inertial forces in the cavity 2, $V_{w 1}=\frac{\pi d_{1}^{2}}{4} S_{1}$ is a liquid volume in the cavity 2, $S_{1}=\frac{S_{h 1}}{2} \cdot\left[(1-\cos \varphi)+\frac{\lambda}{4} \cdot(1-\cos 2 \varphi)\right]+S_{d 1}$ is a current liquid height in the working cavity $2, S_{d l}$ is a linear clearance volume in the pump chamber $1, \Delta p_{i n 1}=-\rho_{w} \frac{d a_{p}}{d \varphi} S_{1} \Delta \varphi$ is a pressure force acting on the liquid in the cavity 2 by reducing the piston 1 acceleration, $\frac{d a_{p}}{d \varphi}=-\frac{S_{h 1}}{2} \omega^{3}(\sin \varphi+2 \lambda \sin 2 \varphi), \Delta V_{w i n p}=\frac{V_{w p}}{E_{w}} \Delta p_{i n p}$ is change in liquid volume due to $S_{2}=S_{\text {2initial }}+\left(S_{h 1}-S_{1}\right) \frac{f_{a}}{f_{2}}-\Delta V_{w \Sigma}$ changes in inertia forces in the pipeline 3 , $V_{w p}=\frac{\pi d_{p}^{2}}{4} l_{p}$ is a liquid volume in the pipeline $3, \Delta p_{\text {inp }}=-\rho_{w} \cdot l_{p} \cdot \frac{f_{a}}{f_{p}} \cdot \frac{d a_{p}}{d \varphi} \cdot \Delta \varphi$ is a pressure force acting on the liquid in the pipeline 3 by varying the piston 1 acceleration, $f_{p}=\frac{\pi d_{p}^{2}}{4}$ is a cross-section area of the pipeline $3, \Delta V_{i n 2}=\frac{V_{w 2}}{E_{w}} \Delta p_{i n 2}$ is a liquid volume variation value in the cavity 4 due to a change in inertia forces, $V_{w 2}=\frac{\pi d_{2}^{2}}{4} S_{2}$ is a liquid volume in the cavity $4, S_{2}=S_{2 \text { initial }}+\left(S_{h 1}-S_{1}\right) \frac{f_{a}}{f_{2}}-\Delta V_{w \Sigma}$ is a liquid height current value in the cavity $4, \Delta p_{i n 2}=-\rho_{w} S_{2} \frac{f_{a}}{f_{2}} \frac{d a_{p}}{d \varphi} \Delta \varphi$ is a pressure force acting on the liquid in the cavity 4 by varying the piston 1 acceleration, $f_{2}=\frac{\pi d_{2}^{2}}{4}$ is a cross-section area of the cavity $4, S_{2 i n i t i a l}$ is a liquid height initial value. 
An elementary change in the liquid volume due to the change of the friction forces can be expressed as:

$$
\Delta V_{w f r}=\Delta V_{w l}+\Delta V_{w \varsigma} \text {, }
$$

where $\Delta V_{w l}$ is an elementary change in the liquid volume due to the change of the resistive forces acting by the full length, $\Delta V_{w \zeta}$ is an elementary change in the liquid volume due to the change of the local resistive forces.

Value $\Delta V_{w l}$ can be expressed as:

$$
\Delta V_{w l}=\Delta V_{w l 1}+\Delta V_{w l 2}+\Delta V_{w l 3}
$$

where $\Delta V_{w l 1}=\frac{V_{w 1}}{E_{w}} \Delta p_{c 1}$ is a liquid volume variation value in the cavity 2, $\Delta p_{c 1}=\rho_{w} g\left(\Delta h_{l 1 i}-\Delta h_{l 1(i-1)}\right), \Delta h_{l 1 i}=\lambda_{l 1 i} \frac{S_{1 i}}{d_{1}} \frac{v_{r i}^{2}}{2 g}$ is a head loss due to resistance along the length in the cavity 2 at the angle of rotation $\varphi_{i}, \Delta h_{l 1(i-1)}=\lambda_{l 1(i-1)} \frac{S_{1(i-1)}}{d_{1}} \frac{v_{r(i-1)}^{2}}{2 g}$ is a head loss due to resistance along the length in the cavity 2 at the angle of rotation $\varphi_{(i-1)}, \lambda_{l 1 i}, \lambda_{l 1(i-1)}$ are friction coefficients along the length in the cavity 2 at the angles of rotation $\varphi_{i}$ and $\varphi_{(i-1)}$, their values are different, because of piston velocity different values, $g$ is a the gravity acceleration, $\Delta V_{w l 2}=\frac{V_{w p}}{E_{w}} \Delta p_{c p}$ is a variation of a liquid volume in the pipeline 3 , $\Delta p_{c p}=\rho_{w} g\left(\Delta h_{l p i}-\Delta h_{l p(i-1)}\right), \Delta h_{l p i}=\lambda_{l p i} \frac{l_{p}}{d_{p}} \frac{v_{p i}^{2}}{2 g}$ is a head loss in the pipeline 3 due to resistance along the length at the angle of rotation $\varphi_{i}, l_{p}$ is the length of the pipeline $3, d_{p}$ is the diameter of the pipeline $3, v_{p i}=\frac{v_{a} f_{p}}{f_{c s}}$ is a liquid velocity in the pipeline at the crankshaft angle of rotation $\varphi_{i}, f_{c s}=\frac{\pi d_{p}^{2}}{4}$ is a cross-section area of the pipeline 3, $\Delta h_{l p(i-1)}=\lambda_{l p(i-1)} \frac{l_{p}}{d_{p}} \frac{v_{a(i-1)}^{2}}{2 g}$ is a head loss in the pipeline 3 due to resistance along the length at the angle of rotation $\varphi_{(i-1)}, v_{p(i-1)}=\frac{v_{a(i-1)} f_{a}}{f_{c s}}$ is a liquid velocity in the pipeline at the crankshaft angle of rotation $\varphi_{(i-1)}, \lambda_{l p i}, \lambda_{l p(i-1)}$ are friction coefficients along the length in the pipeline at the angles of rotation $\varphi_{i}$ and $\varphi_{(i-1)}, \Delta V_{w l 3}=\frac{V_{w 2}}{E_{w}} \Delta p_{c 2}$ is a variation of a liquid volume in the working cavity, $\Delta p_{c 2}=\rho_{w} g\left(\Delta h_{l 2 i}-\Delta h_{l 2(i-1)}\right), \Delta h_{l 2 i}=\lambda_{l 2 i} \frac{S_{2 i}}{d_{2}} \frac{v_{a i}^{2}}{2 g} \frac{f_{a}^{2}}{f_{2}}$ is a head loss due along the length in the chamber 4 at the angle of rotation $\varphi_{i}$, 
$\Delta h_{l 2(i-1)}=\lambda_{l 2(i-1)} \frac{S_{2(i-1)}}{d_{2}} \frac{v_{a(i-1)}^{2}}{2 g}$ is a head loss due along the length in the chamber 4 at the angle of rotation $\varphi_{(i-1)}$.

Two local resistances can be distinguished due to analysing the design circuit presented in Figure 2: a sudden narrowing $\zeta_{2}$ and sudden expansion $\zeta_{1}$.

Value $\Delta V_{w \zeta}$ can be expressed as:

$$
\Delta V_{w \varsigma}=\frac{V_{w}}{E_{w}} \Delta p_{\varsigma}
$$

Value $\Delta p_{\zeta}$ can be expressed as $\Delta p_{\zeta}=\rho_{w} g\left(\Delta h_{\zeta i}-\Delta h_{\zeta(i-1)}\right)$, where $\Delta h_{\zeta i}=\left(\zeta_{1}+\zeta_{2}\right) \frac{v_{p i}^{2}}{2 g}$, $\Delta h_{\zeta(i-1)}=\left(\zeta_{1}+\zeta_{2}\right) \frac{v_{p(i-1)}^{2}}{2 g}$

The values of the local resistance coefficients with a sudden narrowing $\zeta_{2}$ and a sudden expansion $\zeta_{1}$ are determined based on existing recommendations [18].

We obtained a nonlinear equation for $\Delta V_{g}$ by the substitution of the above expressions into the volume conservation equation (6). The solution must be carried out numerically at each angle of rotation of the crankshaft during compression.

Value $\Delta V_{w \Sigma}$ can be expressed as:

$$
\Delta V_{w \Sigma}=\Delta V_{\text {mech }}-\Delta V_{g} .
$$

The decrease in the liquid velocity in cavity 4 can be determined as

$$
\Delta V_{2}=\frac{\Delta V_{w \Sigma}}{f_{2} \Delta \tau}
$$

\section{Results and discussion}

We calculate the liquid compressibility during compression of the two-stage piston hybrid positive-displacement machine with the following initial parameters:

- $S_{h 1}=0.05 m ; d_{1}=0.05 m$;

- $l_{p}=0.05 m ; d_{2}=0.05 m$;

- $d_{p}=0.018 m ; S_{2 \text { initial }}=0$;

- $\lambda=0.2 ; p_{\text {suc }}=2.0 \mathrm{MPa}$;

- $n_{r p m}=500 \mathrm{rp} / \mathrm{min} ; n=1.3$.

As independent variables, it is advisable to choose the following variables during a numerical experiment: $\varepsilon_{d}=p_{d i s} / p_{\text {suc }} ; n_{r p m} ; l_{p}$.

These variables have the greatest effect on the liquid compressibility based on an analysis of the physical processes that take place.

We choose the ratio $\Delta S_{2} / S_{2}$ and the ratio $\Delta v_{2} / v_{2}$ as the response functions, where $\Delta S_{2}$ is the decrease in the liquid height at the end of the compression process in the cavity 4 due to compressibility; $\Delta v_{2}$-reduction of the fluid velocity at the end of the compression process 
in the cavity $4, S_{2}$ - the liquid height at the end of the compression process; $v_{2}$ is the liquid velocity at the end of the compression process.

Water and air are used as working bodies. The planning of the numerical experiment was carried out according to the classical plan with fractional factorial design.

\subsection{Influence of the gas forces}

The gas pressure in the compression process is the main force providing liquid compression in the PHPDM. It is necessary to consider the influence of $\varepsilon_{d}$ and $\mathrm{n}$ among the independent variables on the response functions of the relations $\Delta S_{2} / S_{2}$ and $\Delta v_{2} / v_{2}$.

Figure 3 shows a decrease in the response functions by the increase of pressure ratio $\varepsilon_{d}$.

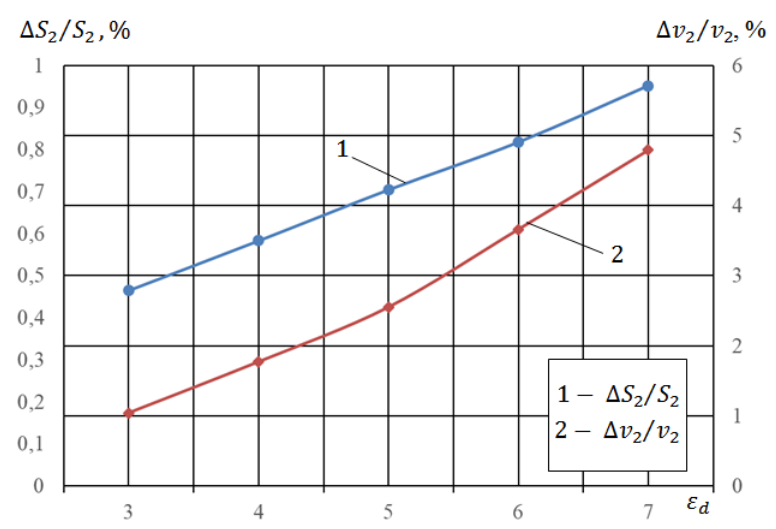

Fig. 3. Dependence of the liquid layer height (1) relative decrease and the liquid velocity (2) relative decrease to the pressure ratio.

The response function values increase at $\varepsilon_{d}=7$ with an increase of $\varepsilon_{d}$ (discharge pressure is $14 \mathrm{MPa}$ ). The relative decrease in the liquid layer height is almost $1 \%$. The decrease in piston velocity at the end of the compression process is $5 \%$. It should be noted that these dependencies are close to linear. We observe an increase in the ratio $\Delta S_{2} / S_{2}$ and a decrease in the ratio $\Delta v_{2} / v_{2}$ with an increase in the polytropic index of the compression process (figure 4).

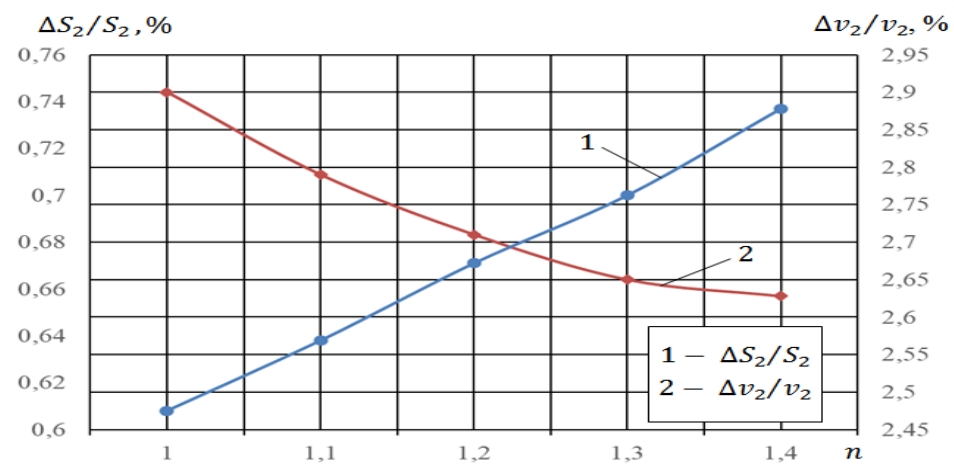

Fig. 4. Dependence of the liquid layer height (1) relative decrease and the liquid velocity (2) relative decrease to the polytropic index of compression. 
The decreasing curve $\Delta S_{2} / S_{2}$ is close to linear from $n$. The change curve $\Delta v_{2} / v_{2}$ is nonlinear from $n$ and the absolute values of the change are insignificant. So the change in $\Delta v_{2} / v_{2}$ is about $0.3 \%$ with a change in $n$ from 1 to 1.4 .

The increase in $\Delta S_{2} / S_{2}$ with an increase in $n$ is explained by the fact that the value of $S_{2}$ decreases almost at a constant value of $\Delta S_{2}$. The decrease in $S_{2}$ is due to the fact that the curve of the compression process goes steeper with increasing $n$, i.e. gas compression is faster. The velocity $v_{2}$ value increases with an almost constant value $\Delta v_{2}$ with a faster compression of the gas. The increase in $v_{2}$ is due to the fact that after $\varphi \geq 3 / 2 \pi$ the piston 1 velocity with adiabatic compression becomes greater than with isothermal.

\subsection{Influence of the inertial forces}

The inertial forces depend on the accelerations of various fluid masses located in the cavities 2 and 4, as well as in the pipeline 3. The accelerations of these masses directly depend on the piston 1 acceleration. The magnitude of the piston 1 acceleration depends on the angular velocity squared. Thus, we consider the dependence of the response functions on the crankshaft revolutions number. These dependencies are presented in figure 5 .

We observe a slight decrease in the functions $\Delta S_{2} / S_{2}$ and $\Delta v_{2} / v_{2}$ with a revolutions number increase. The response functions decrease is since the inertial forces and gas pressure forces are practically in the antiphase. The piston maximum acceleration is achieved almost at the lower point and the gas pressure forces at this time are negligible. The liquid is compressed under the action of inertia. The amount of decrease in the liquid volume exceeds the change in volume due to the piston displacement $V_{\text {mech }}$.

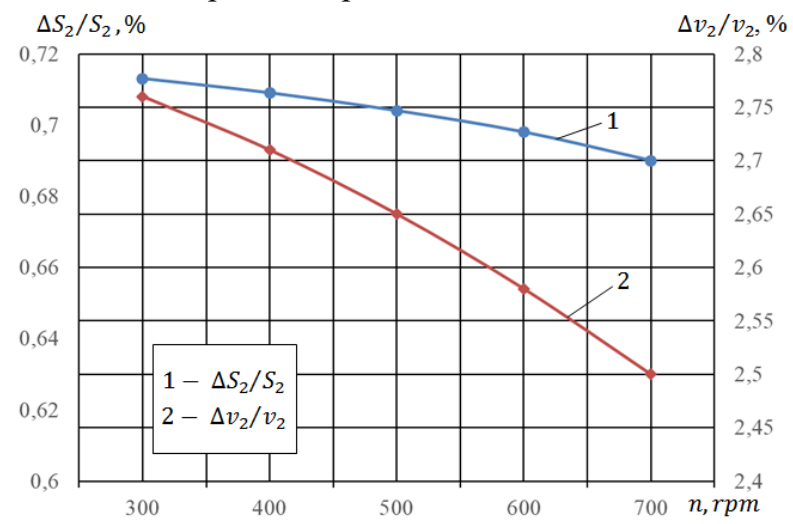

Fig. 5. Dependence of the liquid layer height (1) relative decrease and the liquid velocity (2) relative.

In this case, the compressible gas volume increases by $\Delta V_{g}$, which can be expressed as:

$$
\Delta V_{g}=\Delta V_{w i n}+\Delta V_{w p}-\Delta V_{w c}
$$

The gas total volume in the cavity 4 increases and we observe the gas expansion with decreasing:

$$
p_{g i}=p_{s u c}\left(\frac{V_{s u c}}{V_{s u c}+\Delta V_{g i}}\right)^{n}
$$

where $p_{g i}$ is the gas pressure in the cavity $4, p_{s u c}$ is the suction gas pressure in the cavity 4 , $v_{s u c}$ is the gas volume at the beginning of the compression in the cavity 4 . 
Gas compression starts at the moment when $\Sigma \Delta V_{g i}$ becomes equal to zero. In this case, the initial pressure of the compressible gas will be less than $p_{s u c}$. Besides, the piston acceleration changes its sign at the angle of rotation $\varphi \geq 3 / 2 \pi$, and the inertial forces will prevent liquid compression. The values of $\Delta S_{2}$ and $\Delta v_{2}$ will decrease, which leads to a decrease in the response functions. The change in the response functions with an increase in $n_{r p m}$ is not so significant as with a change in $\varepsilon_{d}$.

\subsection{Liquid volume and resistive force}

The increase in liquid volume and resistive forces are inextricably linked with the increase in the pipeline length.

An increase in the pipeline length $l_{p}$ also leads to an increase in inertial forces. We investigated the response functions of the pipe length.

The values of the response functions increase with the increasing pipe length (figure 6). This increase is very significant and the studied dependencies are close to linear. Since, the value of $\Delta S_{2} / S_{2}$ is $0.429 \%$ at $l_{p}=0.3 \mathrm{~m}$ then it is already $0.969 \%$ at $l_{p}=0.7 \mathrm{~m}$ i.e. more than 2 times. The dependence $\Delta v_{2} / v_{2}$ also behaves similarly. An increase in the response functions with an increase in $l_{p}$ is primarily associated with an increase in the liquid volume $V_{w}$.

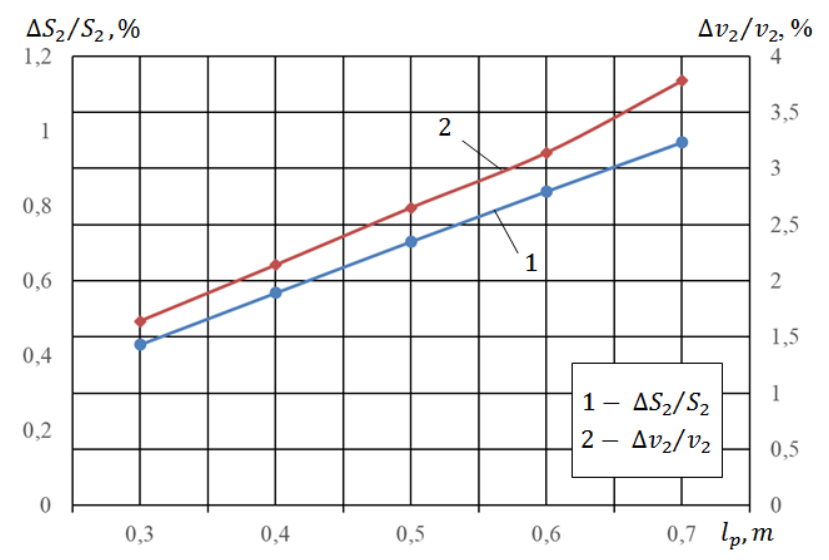

Fig. 6. Dependence of the liquid layer height (1) relative decrease and the liquid velocity (2) relative decrease to the pipeline length.

We can conclude by summing up the parametric analysis that:

- $\varepsilon_{d}$ and $l_{p}$ have the greatest impact on the objective functions;

- then the polytropic index of the compression $n$;

- and the least influence is the crankshaft revolutions number $n_{r p m}$.

\subsection{Analysis of the liquid compressibility influence on working processes and the second compressor stage integral parameters}

In $[19,20]$, a definition is made of the dependence of the thermodynamically effective law of changing the volume of the piston compressor working cavity. It is expressed for the implementation of isothermal and polytropic compression processes. These studies have established that the piston velocity should be maximum at the beginning of the compression process, and then it should decrease. This requirement is not feasible for a piston with a crank drive. The piston velocity decreases with increasing pressure due to the liquid compressibility when using a liquid piston. It brings the compression process closer to a thermodynamically 
effective one. And it leads to an increase in the indicated thermal efficiency of the compressor.

The liquid compressibility leads to a decrease in head loss during the discharge process, especially at its beginning. However, in addition to advantages, the liquid compressibility has drawbacks. They consist of the appearance and increase of dead space in the working compressor chamber. This leads to a decrease in the volumetric coefficient and the compressor delivery rate as a whole.

Thus, the decrease in the delivery rate will be $11.5 \%$ with the liquid compressibility by $1 \%$ at $\varepsilon_{d}=p_{d i s} / p_{\text {suc }}=7$ and the polytropic index of the expansion is 1.3 .

\section{Conclusion}

We have developed a methodology for calculating liquid compressibility in the working processes of the two-stage piston hybrid positive-displacement machine. The action of gas forces, inertial forces, and friction forces on a liquid were taken into account.

As a result of numerical analysis, the relative liquid compressibility is within $1 \%$ during compression, and the relative decrease in velocity is within $5 \%$. Besides, the following parameters have an effect on the liquid compressibility in the order of their influence: the degree of pressure ratio in the stage; pipeline length; polytropic index; crankshaft revolutions number.

The liquid compressibility makes it possible to bring the gas compression process closer to a thermodynamically effective one. But it can lead to a decrease in compressor performance.

\section{References}

1. V. E. Shcherba, Bolshtyanskii A P, Shalai V V and Khodyreva E V, Pump Compressors. Working Processes and Design Fundamentals [in Russian], Mashinostroenie, Moscow, 388 (2013)

2. V. E. Shcherba, Bolshtyanskii A P, Rybak A T, et al. Constructive schemes of hybrid machines of volumetric action [in Russian], Omskiy Nauchniy Vestnik, 1, 2018, pp 1018

3. V. E. Shcherba, Bolshtyanskii A P, Kaigorodov S Y, et al. Benefits of integrating displacement pumps and compressors. Russ. Engin. Res. 36, 2016, pp 174-178

4. A. M. Bazhenov, Shcherba V E, Shalai V V, et al. Modeling the Operation of BulkAction Hybrid Piston Power Systems. Russ. Engin. Res. 39, 2019, pp 377-382

5. V. E. Shcherba, Shalai V V, Nosov E Y, et al. The Operation Analysis of a Reciprocating Hybrid Power Machine with a Gas Volume on Suction Using Various Cooling Liquids, in Proceedings of the 12th International Scientific and Technical Conference "Dynamics of Systems, Mechanisms and Machines", 2018, Omsk; Russian Federation

6. V. E. Shcherba, Shalai V V, Trukhanova D A, et al. Development and experimental study of a piston hybrid energy machine with gas intake volume, Vestnik Mashinostroeniya [In Russian], 4, 2019, pp 18-22

7. A. M. Bazhenov, Development and research of the piston hybrid power machine with stepwise type gap seals: abstract. diss. cand. tech. sciences [In Russian].(2017) Omsk: OmSTU

8. V. E. Shcherba, Tegzhanov A S, Nosov E Y, et al. Comparative Analysis of MassDimensional Indicators of Crossheadless and Crosshead Piston Hybrid Energy Positive 
Displacement Machines, Izv. Vyss. Ucheb. Zaved. Mashinostroenie [In Russian], 9, 2019, pp 88-95

9. V. N. Martynov, Development and research of tubing units for compressing gases and gas-liquid mixtures: abstract. diss. cand. tech. sciences, Moscow, 24, 2009

10. V. E. Shcherba, Shalai V V, Zanin A V, et al. Analysis of Liquid-Heating Process in the Course of Compression in the Working Cavity of the Compressor Section of a Hybrid Piston Power Machine. Chem Petrol Eng 55, 2019, pp 562-577

11. A. V. Zanin, Shcherba V E, et al, Development and Research of an Experimental Prototype of the Positive Displacement Two-Stage Piston Hybrid Energy-Generating Machine, J. Phys.: Conf. Ser. 1441, 012129, 2020

12. V. E. Shcherba, Zanin A V, Nosov E Y, et al. Calculation of Compression and Discharge Processes in a Two-Stage Hybrid Piston Power Machine with Liquid Piston. Chem Petrol Eng 55, 2019, pp 632-641

13. V. E. Shcherba, Shalai V V, Pustovoi N V, et al. On profiling of the working cavity of the second stage in a hybrid piston volumetric power machine. Chem Petrol Eng 56, 2020, pp 125-136

14. Shcherba V E, Pavlyuchenko E A and Kuzhbanov A K 2012 Mathematical Modeling of Processes of Suction and Discharge in a Displacement Pump with Gas Damper. Vestnik Samarskogo Gos. Aero. Uni., 3(34), pp 133-138

15. Shcherba V E, Kuzhbanov A K, Pavlyuchenko E A, et al. 2013 Mathematical model of working processes of reciprocating pump compressor with gas damper [In Russian], Omsk. Nauch. Vestn., Izd. OmSTU, Omsk, 1(117), pp 82-87

16. Shcherba V E, Pavlyuchenko E A and Kuzhbanov A K 2013Mathematical Modeling of Processes of Suction and Discharge in a Displacement Pump with Gas Damper. Chem Petrol Eng 49, 460-466

17. Shcherba V E, Pavlyuchenko E A, Kuzhbanov A K 2014 Parametric Analysis of Operation of Pumping Section of Reciprocating Pump Compressor with Gas Damper. Chem Petrol Eng 50, pp 33-37

18. Altshul A D and Kiselev P G 1975 Hydraulics and aerodynamics. Stroyizdat, Moscow, p 327

19. Shcherba V E 2008 Functional Processes in Positive-Displacement Compressors [in Russian], Nauka, Moscow, p 319

20. Shcherba V E, Shalai V V and Zanin A V 2019 Thermodynamic Foundations of Working Processes in Positive-Displacement Machines Designed for Compression of Two-Phase, Two-Component Working Fluids. Chem Petrol Eng 55, pp 463-472 